\title{
DENTAL ARCH DIMENSIONS IN SURGICALLY REPAIRED UNILATERAL CLEFT LIP AND PALATE OF EGYPTIAN CHILDREN BY OSLO PROTOCOL
}

\author{
Baraka $\mathrm{M}^{t} B D S$. Hanno $\mathrm{A}^{2} B D S, M S c, P h D$. Bakry $\mathrm{N}^{2} B D S, M S c, P h D$. Medra $\mathrm{A}^{3} B D S, M S c, P h D$. \\ Moussa $\mathrm{H}^{4} B D S, M S c, P h D$
}

\begin{abstract} factors resulting in various forms of malocclusion mostly affecting maxillary dental arch. dental study casts. children. arch width/ Oslo protocol.

1. Demonstrator at the Pediatric Dentistry Department, Faculty of Dentistry, Alexandria University, Egypt.

2. Professor of pediatric dentistry, Faculty of Dentistry, Alexandria University, Egypt.

3. Professor of maxilla-facial and plastic surgery, Faculty of Dentistry, Alexandria University, Egypt.
\end{abstract}

INTRODUCTION: Non-syndromic cleft lip and palate is a complex trait caused by the interaction of genetic and environmental

AIM OF THE STUDY: The purpose of this study was to evaluate and compare the dental arch dimensions in surgically repaired unilateral cleft lip and palate children according to Oslo protocol with those of healthy matching non-cleft children in Egypt.

MATERIALS AND METHODS: Comparative cross-sectional study design was used. Thirty-one non-syndromic children with repaired unilateral cleft lip and palate (UCLP), aging 4-9 years, and fulfilling the selection criteria together with thirty-one healthy, matched, non-cleft children (control group) were recruited from Faculty of dentistry, Alexandria University. Both groups were divided into three age groups; 4-5 years, 6-7 years and 8-9 years. For each subject, dental arch dimensions were measured from the

RESULTS: Mean maxillary arch depth and inter-canine arch width were significantly smaller in UCLP children than in non-cleft children in the age groups 6-7 and 8-9 years. Mean inter-molar arch width was not significantly narrower in UCLP children from that in non-cleft children. Mean mandibular arch dimensions of UCLP children did not differ significantly from those of non-cleft

CONCLUSIONS: Children with UCLP, aging 6-9 years old, revealed significant reduction in mean maxillary arch dimensions when compared to healthy matching non-cleft children except for inter-molar arch width which showed no significant reduction.

KEYWORDS: Unilateral cleft lip and palate/ Maxillary arch dimensions/ Mandibular arch dimensions/ Dental arch depth/ Dental

\section{INTRODUCTION}

Cleft lip and cleft palate are among the most common congenital defects in the cranio-facial region. They result from incomplete fusion of maxillary and intermaxillary processes during development of the fetus (1). The etiology of these defects is considered multifactorial through interaction of both genetic and environmental factors. Thus, it can occur as an isolated condition, or as one component of an inherited disease or syndrome (2-4).

The prevalence of cleft lip and palate worldwide is about one per $500-700$ of all births. It differs with cleft type, gender and ethnic origin (5). Most studies give a ratio varying between unilateral and bilateral cleft lips to be predominantly favoring unilateral cleft lips (6-8). Recent data on birth defects from population-based studies originating from Middle East are lacking (9). Meanwhile, few published articles give a rough idea about the incidence of cleft in the region. The overall incidence rate of cleft lip and palate per 1000 live births was 1.5 in Oman in 2001 (10) and 0.9 in Sudan in 2005(11).

According to Athanasiou et al 1987 (12) and many others (13-17), maxillary arch dimensions are generally reduced in patients with clefts. In addition, the primary surgical repairs affect maxillary arch dimensions in children with clefts.

Different surgical protocols and infant orthopedics are used to correct unilateral cleft lip and palate (UCLP). However, there is no general consensus on the optimal method of treatment (18). Some centers use a multistage approach, in which more than one operation is done to close the UCLP and others use one-stage repair approach (19). In order to assess the early treatment outcome regarding dental arch dimensions of these various surgical protocols, different methods have been proposed. Some have taken measurements directly from dental study casts, $(22,23)$ or have used photocopies of models (24).

The ultimate aim of UCLP treatment is to achieve a normalization of functions such as speech, growth of the naso-maxillary complex, arch dimensions and occlusion as well as facial appearance which is a multidisciplinary task (25). The pediatric dentists have a responsibility towards the overall dental care of these children. They are often involved in the presurgical and postsurgical phase of maxillary orthopedics as numerous dental anomalies and malocclusions are encountered during the late primary and mixed dentition stage with UCLP. These malocclusions are either attributed to the congenital clefting itself or may be secondary to the surgical correction of the primary defects. Pediatric dentists can use both active and passive appliances to bring the cleft segments into a more ideal alignment and thereby promote a better initial surgical outcome (26).

In order to identify and implement the highest possible standards of care for UCLP children by the pediatric dentist, assessment of early treatment outcome after primary surgical repair of lip and palate regarding arch dimensions is essential. Although the dental arch dimensions of these children have been evaluated in previous studies (12-17), few investigators in Egypt 
tackled this issue (27). Due to racial differences in development of dental arch and growth patterns, as stated by Lavelle 1975 (28), the scarcity of data in Egypt addressing this unfortunate group of children presents a gap that impedes the delivery of proper dental care to them. The present study aims at filling this gap by highlighting the main characteristics of dental arch dimensions in surgically repaired UCLP children by the Oslo surgical protocol and compares them with those of healthy, matching, non-cleft children to better meet the needs of this vulnerable group of children.

\section{MATERIALS AND METHODS}

The study was a comparative cross sectional study design. Five hundred cleft children registered in both Orthodontics and Maxilla-facial and Plastic Surgery Departments, Faculty of Dentistry, Alexandria University, during the period from September 2014 to April 2015, were examined. Only 31 unilateral cleft lip and palate (UCLP) children, (21 boys and 10 girls) with a mean age of 7.35 years, were suiting the inclusion criteria and were recruited in the study. Another thirtyone healthy, matching, non-cleft children ( 20 boys and 11 girls) with a mean age of 7.13 years were also recruited in the study to serve as the control group. They were either neighbors or school-mates (companions) of UCLP children.

The inclusion criteria of the UCLP children were children of both genders, aging from four to nine years old, with surgically repaired UCLP according to the Oslo surgical protocol (19). (Table 1) Children with any systemic diseases, intellectual disabilities or syndromes and congenital anomalies other than UCLP were excluded from the study as well as UCLP children with previous naso-alveolar moulding, orthodontic treatment or bone graft.

Table 1: Summary of the Oslo surgical protocol.

\begin{tabular}{|c|c|}
\hline & Oslo surgical protocol \\
\hline PSOT $^{*}$ & No \\
\hline 3 months & Lip(Millard) and hard palate closure (single-layer vomer flap) \\
\hline 18months & Soff palate closure (modified von Langenbeck) \\
\hline 8-12 ycars & Alveolarbone grafting \\
\hline
\end{tabular}

* PSOT=presurgical orthopedic treatment

As regards to the non-cleft children (control group), healthy children free from any systemic diseases or syndromes, aging from four to nine-year old, free from oral habits and with limited or no crowding of teeth and no premature loss of teeth were included in this study.

Unilateral cleft lip and palate or non-cleft children with more than one year $( \pm)$ difference between chronological and dental ages (assessment of dental age was based on the Schour and Massler tables) (29) were also excluded. The included children were divided into three age groups, primary dentition group (4-5 years) and two mixed dentition groups (6-7 years) and (8-9 years) since shedding of the mandibular primary canine and growth spurt may occur in the age period 8-9 years (30).

Ethical approval for the study was first obtained from Dental Research Ethics Committee, Faculty of Dentistry, and Alexandria University. The children's parents or guardian were asked to sign an informed consent. Participants were assured of the confidentiality of the collected data and that it was used only for research purposes.

Measurements were carried out by the researcher who was trained and calibrated to develop an acceptable intra-examiner consistency in assessing dental arch dimensions. Intraexaminer reliability was done using Kappa test which was 0.877 for all dental arch dimensions.

For each subject, demographic data were recorded and intra-oral examination was done to determine cleft side. Upper and lower alginate impressions were taken and dental arch dimensions were measured using digital boley gauge (ProDent USA digital Caliper 4". Precision digital caliper measures in either inches or millimeters, from 0 to $4 "$ or 0 to $100 \mathrm{~mm}$ to the nearest $0.01 \mathrm{~mm}$, with easy to read LCD display)

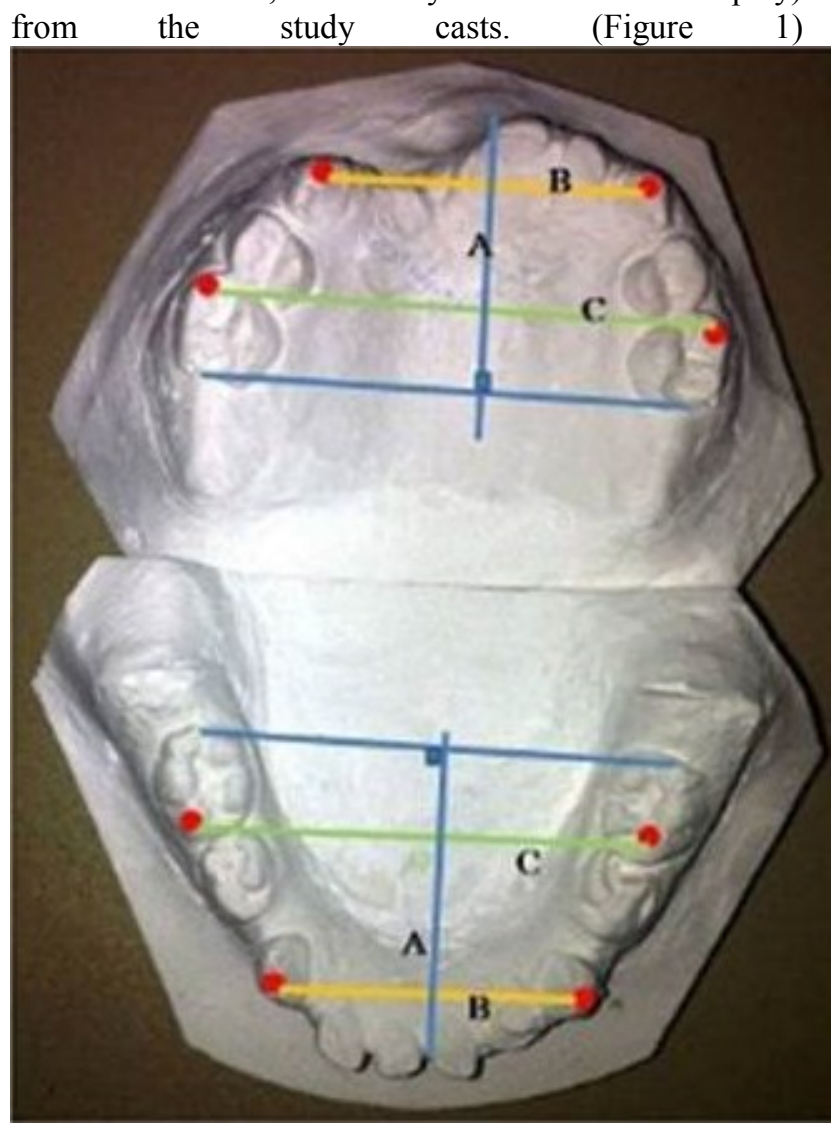

Figure 1: Maxillary and mandibular primary dental arch;

A.Arch depth, B. Inter-canine arch width and

C. Inter-molar arch width

Maxillary and mandibular arch depths were measured in millimeters from the median point between the central incisors (or the mesio-incisal corner of the central incisor in case of missing central incisor) until the tangent line to the distal surface of the second primary molars or mesial surface of the first permanent molars (31).

Maxillary and mandibular inter-canine arch widths were measured in millimeters between cusp tips of the right and left primary or permanent canines in maxillary and mandibular arches respectively. In case of missing or 
un-erupted canines, this measurement was substituted by the inter-first primary molar arch width, which is the distance between buccal cusp tips of the right and left first primary molar in both arches (31).

Maxillary and mandibular inter-molar arch widths were measured in millimeters between mesio-buccal cusp tips of the right and left primary second molars or permanent first molars in maxillary and mandibular arches respectively (31). In cases of cusp tip flattening by wear, the reference point (estimated cusp tip) was marked as the central point in the middle of the wear facet, according to Bishara et al in 1994 (32).

Regarding inter-canine and inter-molar arch widths, for age group 4-5 years, the primary cuspids and primary second molars were used for reference points. As for age group 6-7 years, primary cuspids and permanent first molars were used. For age group 8-9 years, either primary or permanent cuspids were used together with first permanent molars for reference points.

Data were entered into an Excel file using patient identification numbers. Data were fed to the computer and analyzed using IBM SPSS software package version 20.0. Qualitative data presented in the demographic data regarding gender distribution were described using number and percent. Quantitative data were described using range (minimum and maximum), mean, and standard deviation. Values of dental arch dimensions were tested by independent samples ttest. The implemented level of significance was $5 \%$.

\section{RESULTS}

The present study included 62 children, 31 children had unilateral cleft lip and palate (UCLP) and 31 matched non-cleft children. In an attempt to match the two groups, the controls were neighbors and school-mates (companions) of the UCLP children. In the UCLP group, males represented $67.7 \%$ of the group, whereas $32.3 \%$ were females. In the non-cleft group, $64.5 \%$ were males and $35.5 \%$ were females. Their age ranged between 4 and 9 years. The mean age was $(7.35 \pm 1.52$ years $)$ in UCLP children and $(7.13 \pm 1.52$ years $)$ in noncleft children. No statistically significant differences between the two groups regarding gender $(p=0.652)$ or age distribution $(p=0.973)$.

Table 2 shows the comparisons between UCLP and non-cleft children with respect to mean maxillary arch depth. No significant difference existed between both groups in age group 4-5 years $(p=0.476)$. Mean maxillary arch depth was significantly smaller in UCLP children in both age groups 6-7 years $(\mathrm{p}=0.004)$ and $8-9$ years $(\mathrm{p}=0.026)$.

Table 3 shows the comparisons between UCLP and non-cleft children with respect to mean mandibular arch depth. There was no significant difference between groups throughout the studied age groups $(\mathrm{p}=0.124,0.410$ and 0.670 in $4-5$ years, 6-7 years and 8-9 years respectively).

Table 4 shows the comparisons between UCLP and non-cleft children with respect to mean maxillary inter-canine width. There was no significant difference between both groups in the 4-5 years age group $(p=0.180)$. Mean maxillary inter-canine width was significantly narrower in the UCLP group in age groups 6-7 years $(p=0.004)$ and $8-9$ years $(\mathrm{p}=0.001)$.

Table 2: Comparison between UCLP and non-cleft children with respect to mean maxillary arch depth.

\begin{tabular}{|l|cc|ccc|cc|}
\hline & \multicolumn{2}{|c|}{ UCLP } & \multicolumn{3}{|c|}{ Non-cleft } & & \\
\hline Age & $\mathrm{n}$ & Mean $(\mathrm{mm}) \pm$ SD & $\mathrm{n}$ & Mean(mm) \pm SD & $\mathbf{t}$ & $\mathbf{P}$ \\
\hline $4-5$ years & 5 & $22.48 \pm 2.86$ & 5 & $24.18 \pm 1.49$ & 0.7 & 0.476 \\
\hline 6-7years & 11 & $22.38 \pm 3.70$ & 12 & $25.62 \pm 1.94$ & 3.2 & $0.004^{*}$ \\
\hline 8-9 years & 15 & $21.44 \pm 4.39$ & 14 & $25.26 \pm 2.19$ & 2.4 & $0.026^{*}$ \\
\hline
\end{tabular}

$\mathrm{t}$ : independent samples t-test

$* \mathrm{P}<0.05$ (significant)

Table 3: Comparison between UCLP and non-cleft children with respect to mandibular arch depth.

\begin{tabular}{|l|rr|rr|rc|}
\hline & \multicolumn{2}{|c|}{ UCLP } & \multicolumn{2}{|c|}{ Non-cleft } & & \\
\hline Age & $\mathrm{n}$ & \multicolumn{1}{|c|}{ Mean $(\mathrm{mm}) \pm$ SD } & \multicolumn{1}{l|}{ M } & Mean(mm) \pm SD & t & P \\
\hline 4-5 years & 5 & $23.44 \pm 2.19$ & 5 & $21.58 \pm 1.05$ & 1.7 & 0.124 \\
\hline 6-7years & 11 & $24.15 \pm 2.86$ & 12 & $21.44 \pm 2.08$ & 0.8 & 0.410 \\
\hline 8-9 years & 15 & $22.53 \pm 2.33$ & 14 & $22.08 \pm 3.09$ & 0.4 & 0.670 \\
\hline
\end{tabular}

$\mathrm{t}$ : independent samples t-test

Table 4: Comparison between UCLP and non-cleft children with respect to maxillary inter-canine width

\begin{tabular}{|l|lcc|cc|cc|}
\hline & \multicolumn{2}{|c|}{ UCLP } & \multicolumn{2}{|c|}{ Non-cleft } & & \\
\hline Age & n & Mean(mm) \pm SD & n & Mean(mm) \pm SD & t & P \\
\hline 4-5years & 5 & $26.89 \pm 5.15$ & 5 & $30.42 \pm 1.59$ & 1.5 & 0.180 \\
\hline 6-7years & 11 & $26.84 \pm 2.57$ & 12 & $31.71 \pm 2.70$ & 3.2 & $0.004^{*}$ \\
\hline 8-9years & 15 & $26.02 \pm 5.24$ & 14 & $33.98 \pm 2.52$ & 4.7 & $0.001^{*}$ \\
\hline
\end{tabular}

t: independent samples t-test

$* \mathrm{P}<0.05$ (significant)

Table 5 shows the comparisons between UCLP and non-cleft children with respect to mean mandibular inter-canine width. There was no significant difference between groups $(p=0.917$, 0.425 and 0.770 in the age groups of $4-5$ years, 6-7 years and 8-9 years respectively).

Table 5: Comparison between UCLP and non-cleft children with respect to mandibular inter-canine width

\begin{tabular}{|l|rr|rr|rr|}
\hline & \multicolumn{2}{|c|}{ UCLP } & \multicolumn{2}{|c|}{ Non-cleft } & & \\
\hline Age & $\mathrm{n}$ & Mean(mm) \pm SD & $\mathrm{n}$ & Mean(mm) \pm SD & t & P \\
\hline 4-5years & 5 & $23.95 \pm 1.94$ & 5 & $23.94 \pm 0.45$ & 0.1 & 0.917 \\
\hline 6-7years & 11 & $25.98 \pm 2.14$ & 12 & $24.75 \pm 2.97$ & 0.8 & 0.425 \\
\hline 8-9 years & 15 & $26.92 \pm 4.37$ & 14 & $26.22 \pm 2.11$ & 0.3 & 0.770 \\
\hline
\end{tabular}

t: independent samples t-test 
Table 6 shows the comparisons between UCLP and non-cleft children with respect to mean maxillary inter-molar width. There was no significant difference between groups $(\mathrm{p}=0.743$, 0.884 and 0.285 in 4-5 years, 6-7 years and 8-9 years respectively). Table 7 shows the comparisons between UCLP and non-cleft children in different age groups with respect to mean mandibular inter-molar width. There was no significant difference between groups ( $p=0.529$, 0.134 and 0.305 in 4-5 years, 6-7 years and 8-9 years respectively).

Table 6: Comparison between UCLP and non-cleft children with respect to maxillary inter-molar width

\begin{tabular}{|l|rr|rr|rr|}
\hline & \multicolumn{2}{|c|}{ UCLP } & \multicolumn{2}{|c|}{ Non-cleft } & & \\
\hline Age & $\mathbf{n}$ & Mean(mm) \pm SD & n & Mean(mm) \pm SD & t & P \\
\hline 4-5years & 5 & $40.51 \pm 3.27$ & 5 & $42.90 \pm 4.20$ & 0.3 & 0.743 \\
\hline 6-7years & 11 & $45.15 \pm 4.82$ & 12 & $46.15 \pm 3.92$ & 0.1 & 0.884 \\
\hline 8-9 years & 15 & $45.26 \pm 4.99$ & 14 & $49.75 \pm 3.84$ & 1.1 & 0.285 \\
\hline
\end{tabular}

t: independent samples t-test

Table 7: Comparison between UCLP and non-cleft children with respect to mandibular inter-molar width

\begin{tabular}{|l|rr|rr|rr|}
\hline & \multicolumn{2}{|c|}{ UCLP } & \multicolumn{2}{|c|}{ Non-cleft } & & \\
\hline Age & $\mathrm{n}$ & Mean(mm) \pm SD & n & Mean(mm) \pm SD & t & P \\
\hline 4-5years & 5 & $36.27 \pm 2.09$ & 5 & $36.30 \pm 2.56$ & 0.7 & 0.529 \\
\hline 6-7years & 11 & $45.24 \pm 3.23$ & 12 & $45.26 \pm 4.88$ & 1.6 & 0.134 \\
\hline 8-9 years & 15 & $45.37 \pm 3.25$ & 14 & $45.44 \pm 3.97$ & 1.0 & 0.305 \\
\hline
\end{tabular}

$\mathrm{t}$ : independent samples t-test

\section{DISCUSSION}

Children with unilateral cleft lip and palate (UCLP) usually suffer from deficiency in growth of the naso-maxillary complex due to tissue deficiency, scar tissue formation after early reconstructive surgery and inherent growth retardation. Maxillary dental arch development is also retarded due to the naso-maxillary complex deficiency (12).

The surgical outcomes for the early repair of UCLP are highly variable and this can be attributed to several factors (33). This fact, in addition to scarcity of data available in Egypt regarding early outcome assessment initiated this descriptive study to establish baseline information for the pediatric dentists to improve the standards of care available to this vulnerable group of children.

In the present study, UCLP children were divided into three critical age periods; namely primary dentition (4-5 years), two mixed dentitions (6-7 years) and (8-9 years). Among these age groups, different preventive and interceptive orthodontic interventions have been reported to improve the dental arch dimensions and occlusion for UCLP children (33).
A total of thirty one UCLP children (21 males and 10 females) with an age range 4-9 years were included. The mean age scores were $7.35 \pm 1.52$ years in UCLP children and $7.13 \pm 1.52$ years in non-cleft children. Males and females were pooled together the same way as in the normative data.

In the present study, digital calipers were used to measure the dental arch dimensions from dental casts directly. This method was used rather than the visual 3D dental cast analysis on account of the assumption of Ooster Kamp et al in 2006 (34), who reported that the reliability of visual 3D dental cast analysis is uncertain and timeconsuming.

The mean maxillary arch dimensions of UCLP children at age group 4-5 years were not significantly smaller than those of non-cleft children. The present data are in contrast to the reported results that maxillary arch dimensions would have been adversely affected by the scar tissue developed after palatoplasty $(16,35,36)$. This disagreement could be attributed to the fact that the number of UCLP and non-cleft children available in this age group was smaller in the present study.

Similarly the mean maxillary dental arch depth was not significantly smaller in 4-5 year old UCLP children. However, in the age groups 6-7 years and 8-9 years, it was significantly deficient compared to their controls. This is in agreement with the study of Athanasiou et al in 1988 (37). Deficiency could be related to the palate-version of the maxillary central incisors, the mesial drifting of the first permanent molars due to congenitally missing teeth as well as ectopically erupting first permanent molars.

In the age group 4-5 years, the mean maxillary inter-canine width of the UCLP children was smaller than that of non-cleft group, although it did not reach a significant level. This finding disagrees with that reported in the studies performed by Athanasiou et al in 1988 (37) and Stein et al in 2007 (38). The reported data showed that the mean maxillary inter-canine width in the 4-5 year old UCLP children was significantly narrower than in the non-cleft group. Again, the small number of 45 year old children in the present study probably explains this contradiction. On the other hand, the mean maxillary inter-canine width was significantly narrower in UCLP group in the age groups 6-7 years and 8-9 years, which support the findings of the previous studies $(36,37)$.

Mean maxillary inter-molar width values were not significantly different from that of non-cleft group in all age groups. This coincides with the study performed by Garrahy et al in 2005 (16). The reason behind this finding might be due to larger maxillary inter-molar arch width in UCLP children at birth compared to non-cleft children as reported by Kramer et al in 1996 (35). Consequently, after surgical palatal closure and scar tissue formation, the inter-molar arch width values of UCLP children start to approach those of 
non-cleft children during the primary and mixed dentition stages.

In the present study, there were no significant differences in the mean mandibular arch dimensions among all three age groups between UCLP and non-cleft children. The results suggest that the effect of maxillary arch surgery does not influence remarkably on the mandibular arch dimensions. This finding agrees with other studies such as that of Derijcke et al in 1994 (39) and Garrahy et al in 2005 (16).

The present study revealed several limitations that should be taken into consideration in future studies. One of these limitations is the small sample size especially in the $4-5$ year group. The study included attendants of the Maxillo-Facial and Plastic Surgery Department as well as Orthodontic Department, Faculty of Dentistry, Alexandria University during the period from September 2014 throughout April 2015. In spite of the several significant differences between UCLP and their matching controls, these results should still be interpreted cautiously and should not be regarded as evidence based parameters in the Egyptian population.

However, a strength point of this study is the selection of controls that were companions of the UCLP children to match age, gender, socioeconomic variables.

Another strength point is the ability to use linear dimensional analysis for data comparisons which are more reliable than categorizations of dental occlusion using the Five-Year-Old or Goslon indices which use an ordinal scale of five categories, excellent, good, fair, poor, very poor, to identify an individual's occlusal status with a consequent general prediction about the relative complexity of future correction of malocclusion.

In spite of the mentioned limitations, the present study opens channels to further investigate dental arch parameters and relationships in larger samples of surgically repaired UCLP children and other cleft types using surgical protocols other than Oslo technique

\section{CONCLUSIONS}

Based on the results of present study, the authors concluded that children with UCLP revealed significant reduction in mean maxillary arch measurements (maxillary arch depth and intercanine arch width) when compared to healthy matching non-cleft children. Mean maxillary intermolar arch width did not differ significantly among UCLP and non-cleft groups. Mean mandibular arch parameters (arch depth, inter-canine arch width, inter-molar arch width) were not influenced by the deficient maxillary arch and were all comparable to those of non-cleft group.

\section{RECOMMENDATIONS}

Further research should be initiated to assess arch dimensions following different interceptive orthodontic measures and alveolar bone grafting in attempt to improve surgical outcomes. In addition, characteristics of arch dimensions in children with different types of cleft lip and palate in Egyptian children should be also investigated.

\section{CONFLICT OF INTEREST}

The authors declare that they have no conflicts of interest.

\section{REFERENCES}

1. Marazita ML, Mooney MP. Current concepts in the embryology and genetics of cleft lip and cleft palate. Clin Plast Surg. 2004; 31: 125-40.

2. Cobourne MT. The complex genetics of cleft lip and palate. Eur J Orthod 2004; 26: 7-16.

3. Rice DP. Craniofacial anomalies: from development to molecular pathogenesis. Curr Mol Med. 2005; 5: 699-722.

4. Mossey PA, Little J, Munger RG, Dixon MJ, Shaw WC. Cleft lip and palate. Lancet. 2009; 374: 177385.

5. World Health Organization (WHO). Oral Health. Fact Sheet No 38. April 2012. Available from URL: http://www.who.int/mediacentre/factsheets/fs3 18/en/ Accessed on $3^{\text {rd }}$ of March 2015.

6. Abyholm FE. Cleft lip and palate in Norway, I. Registration, incidence and early mortality of infants with cleft lip and palate. Scand J Plast Reconstr Surg. 1978; 12:29-43.

7. Womersley J, Stone DH. Epidemiology of facial clefts. Arch Dis Child 1987; 62:717-20.

8. Jensen BL, Kreiborg S, Dahl E, Fogh-Andersen P. Cleft lip and palate in Denmark, 1976-1981: Epidemiological, variability, and early somatic development. Cleft Palate J. 1988; 25:258-69.

9. World Health Organization (WHO). What is the burden of oral disease? Available from URL:

http://www.who.int/oral_health/disease_burde n/global/en/ Accessed on $3^{\text {rd }}$ of March 2015.

10. Rajab A, Thomas C. Oral clefts in the Sultanate of Oman. Eur J Plast Surg. 2001; 24:230-3.

11. Suleiman AM, Hamzah ST, Abusalab MA, Samaan KT . Prevalence of cleft lip and palate in a hospitalbased population in the Sudan. Int J Paediatr Dent. 2005; 15:185-9.

12. Athanasiou AE, Mazaheri M, Zarrinnia $\mathrm{K}$. Longitudinal study of the dental arch dimensions in hard and soft palate clefts. J Pedod. 1987; 12: 35-47.

13. Nystrom M, Ranta R. Sizes of dental arches and interdental space in 3-year-old children with and without cleft lip/palate. Eur J Orthod. 1989; 11: 82-8.

14. da Silva Filho O, Ramos A, Abdo R. The influence of unilateral cleft lip and palate on maxillary dental arch morphology. Angle Orthod. 1992; 62: 283-90.

15. McCance A, Roberts-Harry D, Sherriff M, Mars M, Houston WJ. Sri Lankan cleft lip and palate study model analysis: clefts of the secondary palate. Cleft Palate Craniofac J. 1993; 30: 227-30. 
16. Garrahy A, Millett DT, Ayoub AF. Early assessment of dental arch development in repaired unilateral cleft lip and unilateral cleft lip and palate versus controls. Cleft Palate Craniofac J. 2005; 42: 385-91.

17. Lewis BR, Stern MR, Willmot DR. Maxillary anterior tooth size and arch dimensions in unilateral cleft lip and palate. Cleft Palate Craniofac J. 2008; 45: 639-46.

18. Costello B, Ruiz R. Cleft lip and palate: Comprehensive treatment planning and primary repair. In: Miloro M, Ghali GE, Larsen PE, Waite P. Peterson's principles of oral and maxillofacial surgery, Second ed. Hamilton: BC Decker inc; 2004: 839-58.

19. Fudalej P, Hortis-Dzierzbicka M, Dudkiewicz Z, Semb G. Dental arch relationship in children with complete unilateral cleft lip and palate following Warsaw (one-stage repair) and Oslo protocols. Cleft Palate Craniofac J. 2009; 46:648-53.

20. Millard RD. Extensions of the rotation-advancement principle for wide unilateral cleft lips. Plast Reconstr Surg. 1968; 42:535-44.

21. Stewart TL, Fisher DM, Olson JL. Modified Von Langenbeck Cleft palate repair using an anterior triangular flap: decreased incidence of anterior oronasal fistulas. Cleft Palate Craniofac J. 2009; 46:299-304.

22. Ross ML. A comparative model analysis of untreated cleft palate adults and normal adults. Am J Orthod. 1962; 48:63-64.

23. Pruzansky S, Aduss H. Prevalence of arch collapse and malocclusion in complete unilateral cleft lip and palate. Rep Congr Eur Orthod Soc. 1967; 43:365-82.

24. Huddart AG, Bodenham RS. Maxillary arch dimensions in normal and unilateral cleft palate subjects. Cleft Palate J. 1969; 6: 471-87.

25. Long RE, Hathaway R, Daskalogiannakis J, Mercado A, Russell K, Cohen M et al. The americleft study: an inter-center study of treatment outcomes for patients with unilateral cleft lip and palate part 1. Principles and study design. Cleft Palate Craniofac J. 2011; 48: 239-43.

26. Jones J, Sadove AM, Dean JA, Huebener DV. Multidisciplinary team approach to cleft lip and palate management. In: McDonald R, Avery D, Dean J. Dentistry for the Child and Adolescent, Ninth ed. Missouri: Mosby; 2011: 550-60.

27. El-Koutby MM, Hafez SA. Prevalence of malocclusion in the primary and early mixed dentition in a group of Egyptian children with complete unilateral cleft lip and palate. Egypt Dent J. 1993; 39:479-84.

28. Lavelle CL. The shape of dental arch. Am J ORthod. 1975; 67: 176-84.

29. Graber TM. Orthodontics, principles and practice. Philadelphia: WB Saunders; 1974: 90-1.

30. Ferguson D J. Growth of the face and dental arches. In: McDonald R, Avery D, Dean J. Dentistry for the Child and Adolescent, Ninth ed. Missouri: Mosby; 2011: 550-60.

31. Burdi AR, Moyers RE. Development of the dentition and the occlusion. In: Moyers RE. Handbook of
Orthodontics, Fourth ed. Chicago: Year Book Medical Publisher Inc; 1988: 99-146.

32. Bishara SE, Bayati P, Zaher AR, Jakobsen JR. Comparisons of the dental arch changes in patients with Class II, division 1 malocclusions: extraction vs nonextraction treatments. Angle Orthod. 1994; 64: 351-8.

33. Reiser E. Cleft Size and Maxillary Arch Dimensions in Unilateral Cleft Lip and Palate and Cleft Palate. PhD Thesis, Faculty of Medicine, Uppsala University, 2011.

34. Oosterkamp BC, van der Meer WJ, Rutenfrans M, Dijkstra PU. Reliability of linear measurements on a virtual bilateral cleft lip and palate model. Cleft Palate Craniofac J. 2006; 43:519-23.

35. Skoog T. Plastic Surgery. New methods and refinements. Stockholm: Almquist \& Wiksell; 1974.

36. Kramer GJ, Hoeksma JB, Prahl-Andersen B. Early palatal changes after initial palatal surgery in children with cleft lip and palate. Cleft Palate Craniofac J. 1996; 33: 104-11.

37. Athanasiou AE, Mazaheri M, Zarrinnia K. Dental arch dimensions in patients with unilateral cleft lip and palate. Cleft Palate J. 1988; 25:139-45

38. Stein S, Dunsche A, Gellrich NC, Härle F, Jonas I. One-or-Two- stage palate closure in patients with unilateral cleft lip and palate: Comparing cephalometric and occlusal outcomes. Cleft Palate Craniofac J. 2007: 44; 13-22.

39. Derijcke A, Kuijpers-Jagtman AM, Lekkas C, Hardjowasito W, Latief B. Dental arch dimensions in unoperated adult cleft palate-palate patients: an analysis of 37 cases. J Craniofac Genet Dev Biol. 1994; 14: 69-74. 